\title{
Patient ethnicity and the risk of immune-mediated adverse drug reactions
}

\author{
Ana Alfirevic ${ }^{*}, 1$ \\ ${ }^{1}$ The Wolfson Centre for Personalised Medicine, Department of Molecular \& Clinical Pharmacology, Institute of Translational \\ Medicine, University of Liverpool, Block A: Waterhouse Buildings, 1-5, Brownlow Street, Liverpool L69 3GL, UK \\ * Author for correspondence: Tel.: +44 151794 5551; Fax: +44 151794 5059; ana.alfirevic@liverpool.ac.uk
}

\section{"Several HLA alleles could be associated with immune reactions to one drug and a range of clinical phenotypes in diverse populations"}

First draft submitted: 17 July 2017; Accepted for publication: 17 July 2017; Published online: 4 October 2017

Keywords: adverse drug reactions $\bullet$ ethnicity $\bullet$ HLA $\bullet$ immune

Immune-mediated adverse drug reactions (ADRs) lead to hospitalization in approximately $9 \%$ of US patients who visit Emergency Departments for adverse drug events [1]. However, most severe immune ADRs are rare, but can be life-threatening. They are categorized as type B or 'off target' reactions that cannot be predicted from the known pharmacological action of the drug that caused it. Immune-mediated ADRs, comprising $<20 \%$ of all ADRs, could be further classified according to Gell and Coombs into immediate (antibody dependent) and delayed (T-cell mediated) reactions. Clinical phenotypes vary considerably; immediate reactions include urticaria, angioedema, bronchospasm and pruritus usually occurring within $1 \mathrm{~h}$ after drug administration; delayed reactions occurring within 6 weeks after drug administration often present with skin symptoms ranging from mild maculopapular exanthema to sometimes severe blistering skin reactions with systemic symptoms such as StevensJohnson syndrome, toxic epidermal necrolysis or acute generalized exanthematous pustulosis. Nomenclature for severe drug-induced cutaneous adverse reactions with systemic symptoms is variable and includes the following terms: drug hypersensitivity syndrome, hypersensitivity syndrome, drug reaction with eosinophilia and systemic symptoms. Organ-specific toxicities such as drug-induced myotoxicity, liver injury, agranulocytosis, kidney or pancreas injury can also include immunological etiology as demonstrated by clinical phenotype characteristics and significant associations with genes with immune function within the MHC on chromosome 6, primarily HLA genes [2-7].

Pathophysiological mechanisms of immune ADRs are not fully understood, however, there are three recognized models that explain T-cell-mediated hypersensitivity [8]. They include: hapten/prohapten model (drug example - sulfamethoxazole), where a small drug or its reactive metabolite induces an immune response by covalently binding to an endogenous protein to become immunogenic and is then presented on an MHC molecule to a T-cell receptor; the pharmacological interactions $(\mathrm{p}-\mathrm{i})$ model proposes that a drug can bind directly to the T-cell receptor or MHC molecule and stimulate T cells directly, independent of antigen processing (drug example carbamazepine); and the altered repertoire model, where a drug binds noncovalently in a concentration-dependent manner to the peptide binding groove of the HLA molecule and changes the chemistry of the HLA molecule that displays altered self-peptides which are recognized by $\mathrm{T}$ cells (drug example - abacavir).

Genetic factors have been implicated in immune-mediated ADRs. Particularly prominent are genetic associations with the HLA alleles. HLA allelic frequencies vary greatly in diverse worldwide populations (Allelefrequency.net database) [9] and there are many population-specific associations described. One of the examples often cited in literature is an association between $H L A-B^{*}$ 15:02 and carbamazepine-induced SJS/TEN in individuals of east Asian ancestry, but not in European or African populations [10,11]. Indeed, data from $>10$ million healthy individuals from the allelefrequency.net database show that this allele is present at a frequency between 2 and $36 \%$ in east Asians, but is extremely rare in other populations ( 1 in 10,000 to 100,000 individuals carry that allele). Therefore, the Clinical Pharmacogenetics Implementation Consortium (CPIC) guidelines [12], recognized by several drug 
regulatory agencies including the US FDA, European EMA and Japanese PMDA, recommend screening for $B^{*}$ 15:02 before starting treatment with carbamazepine only in patients with east Asian ancestry [13]. In contrast, several other HLA risk alleles including $H L A-B^{*} 57: 01, B^{*}$ 58:01 and $A^{*} 31: 01$ seem to predispose individuals of all races/ethnicities to drug-induced hypersensitivity and organ-directed toxicity to a number of medications including an antiretroviral abacavir, antibiotic flucloxacillin, antigout drug allopurinol and an antiepileptic carbamazepine, respectively [2,14-16]. Strong associations between these drug/HLA allele pairs have been demonstrated in genomewide screens of Caucasian, Asian and African populations and in phenotypes of diverse severity.

We and others $[17,18]$ have investigated what is the relevance of ethnic background to immune ADRs and how genetic ancestry can contribute to the diagnosis, prediction or prevention of ADRs [19]. Terminology used to classify human variability in genomics includes race, ancestry and ethnicity. These terms are often used interchangeably, however, the concept of ethnicity includes distinctive national, religious, cultural background and affiliation, while race has biological and observable physical features, implying that race is socially imposed as perceived by others. Ancestry informative markers are used in genetics for precise characterization of individuals' biological ancestry. With increasing number of markers, better precision is achieved in defining fine-scale population structure and interestingly, genetic differentiation shows remarkable concordance with geography [20]. Genetic associations between immune-mediated ADRs and genes with immune function including the most polymorphic HLA genes (HLA-B has $>3800$ documented alleles) is ethnicity-specific for some clinical phenotypes and for some drugs. An association between one $H L A$ allele (such as $B^{*} 15: 02$ ) and hypersensitivity to more than one antiepileptic drugs including carbamazepine (CBZ), lamotrigine, phenytoin has been described. In addition, two or more Several HLA alleles could be associated with immune reactions to one drug and a range of clinical phenotypes in diverse populations. We would highlight several issues that contribute to such diversity in reporting: first, severe drug-induced hypersensitivity is rare and it is difficult to recruit large numbers of patients into studies therefore, majority of papers reporting associations contain a small number of patients, mostly $<100$; second, majority of these associations could not prove causality in subsequent functional analyses and multifactorial mechanisms of toxicity are still unknown for most of the drug-allele pairs; third, the role of HLA allele haplotypes has not been addressed fully as low sample sizes prevent from any meaningful analyses. We endeavored to analyze potential risk haplotypes for drug-induced liver injury using healthy individual HLA haplotype data from worldwide populations in publicly accessible sources and interestingly, narrowed down the number of risk haplotypes shared with several drugs implicated in liver injury [21]; fourth, reporting of HLA associations with adverse drug reactions in populations of African or South American origin is scarce, partly because of the lower number of clinical observational studies being undertaken and partly because technology for genome-wide association analyses has been biased towards selection of markers present at higher frequency in white populations of northern European ancestry.

A substantial investment is required for all worldwide populations to benefit equally from advances in genomic and personalized medicine. Recent advances in sequencing technology enabled the members of the Human Heredity and Health in Africa (H3Africa) consortium (a partnership between NIH, the African Society of Human Genetics and Wellcome Trust) and Illumina to develop an array for genome wide association studies (GWAS), which includes 2.5 million variants of specific interest to African populations. This new array will foster genetic and epidemiological research with the aim to improve health across diverse African populations. Recently, other arrays have become available, specific for Hispanic, African-American or Japanese [22] populations. In addition, further development of GWAS arrays together with increased availability of reference datasets generated by next generation sequencing from diverse populations, may improve HLA imputation methods. Currently, estimated accuracy of four-digit HLA allele imputation from SNPs using $H L A^{*} I M P 2$ varies; for HLA-B locus, for example, estimated accuracy in Europeans is $94 \%$, in Asians $86 \%$, in Africans $82 \%$ and in Latinos $75 \%$. Furthermore, even lower accuracy has been estimated for proxy HLA SNPs to predict risk specific HLA alleles associated with immune ADRs across racial groups. Proxy HLA markers do not work in diverse populations, they are often in incomplete linkage disequilibrium with HLA alleles in some but not all ethnicities. In a mixed population representing African, Caucasian, Hispanic and Asian Pacific Islanders sensitivity and specificity for rs2395029, a proxy SNP for $B^{*} 57: 01$, was close to $100 \%$, however, sensitivity for $B^{*} 15: 02$ and $B^{*} 58: 01$ was approximately $32 \%$ [23]. Meta-analysis of trans-ethnic GWAS studies may help to identify causal genetic risk factors for immune ADRs shared among diverse ethnicities. Several novel models are being developed which will improve power and increase speed of trans-ethnic GWAS analyses. Such new computational approaches to large dataset analyses are required to help addressing additional complexities of the human genome in diverse populations including structural variants [24]. 
Clinical implementation of screening programmes in prevention, diagnosis and management of immune ADRs should take into consideration variability concerning diverse ethnicities in cost-effectiveness [25], availability of genotyping, incidence of ADRs and training of healthcare professionals and patient population. Although recent international efforts have significantly improved health inequalities and availability of genomic knowledge and technologies across all continents, further work needs to be done to improve safety of medicines in individuals of all ethnic background who may benefit from personalization of treatment.

Financial \& competing interests disclosure

The author has no relevant affiliations or financial involvement with any organization or entity with a financial interest in or financial conflict with the subject matter or materials discussed in the manuscript. This includes employment, consultancies, honoraria, stock ownership or options, expert testimony, grants or patents received or pending, or royalties.

No writing assistance was utilized in the production of this manuscript.

\section{References}

1 Shehab N, Lovegrove MC, Geller AI, Rose KO, Weidle NJ, Budnitz DS. US emergency department visits for outpatient adverse drug events, 2013-2014. JAMA 316(20), 2115-2125 (2016).

2 Daly AK, Donaldson PT, Bhatnagar P et al. HLA-B*5701 genotype is a major determinant of drug-induced liver injury due to flucloxacillin. Nat. Genet. 41(7), 816-819 (2009).

3 Goldstein JI, Jarskog LF, Hilliard C et al. Clozapine-induced agranulocytosis is associated with rare $H L A-D Q B 1$ and $H L A-B$ alleles. Nat. Comm. 5, 4757 (2014).

4 Heap GA, So K, Weedon M et al. Clinical features and HLA association of 5-aminosalicylate (5-ASA)-induced nephrotoxicity in inflammatory bowel disease. J. Crohn's Colitis 10(2), 149-158 (2016).

5 Heap GA, Weedon MN, Bewshea CM et al. HLA-DQA1-HLA-DRB1 variants confer susceptibility to pancreatitis induced by thiopurine immunosuppressants. Nat. Genet. 46(10), 1131-1134 (2014).

6 Mammen AL, Gaudet D, Brisson D et al. Increased frequency of $D R B 1^{*}$ 11:01 in anti-hydroxymethylglutaryl-coenzyme A reductase-associated autoimmune myopathy. Arthritis Care Res. (Hoboken) 64(8), 1233-1237 (2012).

7 Zhang FR, Liu H, Irwanto A et al. HLA-B*13:01 and the dapsone hypersensitivity syndrome. N. Engl. J. Med. 369(17), 1620-1628 (2013).

8 Cho T, Uetrecht J. How reactive metabolites induce an immune response that sometimes leads to an idiosyncratic drug reaction. Chem. Res. Toxicol. 30(1), 295-314 (2017).

9 Gonzalez-Galarza FF, Takeshita LY, Santos EJ et al. Allele frequency net 2015 update: new features for HLA epitopes, KIR and disease and HLA adverse drug reaction associations. Nucleic Acids Res. 43(Database issue), D784-D788 (2015).

10 Alfirevic A, Jorgensen AL, Williamson PR, Chadwick DW, Park BK, Pirmohamed M. HLA-B locus in Caucasian patients with carbamazepine hypersensitivity. Pharmacogenomics 7(6), 813-818 (2006).

11 Chung WH, Hung SI, Hong HS et al. Medical genetics: a marker for Stevens-Johnson syndrome. Nature 428(6982), 486 (2004).

12 Whirl-Carrillo M, Mcdonagh EM, Hebert JM et al. Pharmacogenomics knowledge for personalized medicine. Clin. Pharmacol. Ther. 92(4), 414-417 (2012).

13 Leckband SG, Kelsoe JR, Dunnenberger HM et al. Clinical Pharmacogenetics Implementation Consortium guidelines for HLA-B genotype and carbamazepine dosing. Clin. Pharmacol. Ther. 94(3), 324-328 (2013).

14 Mallal S, Phillips E, Carosi G et al. HLA-B*5701 screening for hypersensitivity to abacavir. N. Engl. J. Med. 358(6), 568-579 (2008).

15 Mccormack M, Alfirevic A, Bourgeois S et al. HLA-A*3101 and carbamazepine-induced hypersensitivity reactions in Europeans. N. Engl. J. Med. 364(12), 1134-1143 (2011).

16 Saito Y, Stamp LK, Caudle KE et al. Clinical Pharmacogenetics Implementation Consortium (CPIC) guidelines for human leukocyte antigen B (HLA-B) genotype and allopurinol dosing: 2015 update. Clin. Pharmacol. Ther. 99(1), 36-37 (2016).

17 Chan SL, Jin S, Loh M, Brunham LR. Progress in understanding the genomic basis for adverse drug reactions: a comprehensive review and focus on the role of ethnicity. Pharmacogenomics 16(10), 1161-1178 (2015).

18 Ghattaoraya GS, Middleton D, Santos EJ, Dickson R, Jones AR, Alfirevic A. Human leucocyte antigen-adverse drug reaction associations: from a perspective of ethnicity. Int. J. Immunogenet. 44(1), 7-26 (2017).

19 Alfirevic A, Pirmohamed M. Genomics of adverse drug reactions. Trends Pharmacol. Sci. 38(1), 100-109 (2017).

20 Leslie S, Winney B, Hellenthal G et al. The fine-scale genetic structure of the British population. Nature 519(7543), 309-314 (2015).

21 Alfirevic A, Gonzalez-Galarza F, Bell C et al. In silico analysis of HLA associations with drug-induced liver injury: use of a HLA-genotyped DNA archive from healthy volunteers. Genome Med. 4(6), 51 (2012). 
22 Ueta M, Sawai H, Shingaki R et al. Genome-wide association study using the ethnicity-specific Japonica array: identification of new susceptibility loci for cold medicine-related Stevens-Johnson syndrome with severe ocular complications. J. Hum. Genet. 62(4), 485-489 (2017).

23 He Y, Hoskins JM, Clark S et al. Accuracy of SNPs to predict risk of HLA alleles associated with drug-induced hypersensitivity events across racial groups. Pharmacogenomics 16(8), 817-824 (2015).

24 Sudmant PH, Rausch T, Gardner EJ et al. An integrated map of structural variation in 2,504 human genomes. Nature 526(7571), 75-81 (2015).

25 Kapoor R, Martinez-Vega R, Dong D et al. Reducing hypersensitivity reactions with $H L A-B^{*} 5701$ genotyping before abacavir prescription: clinically useful but is it cost-effective in Singapore? Pharmacogenet. Genomics 25(2), 60-72 (2015). 